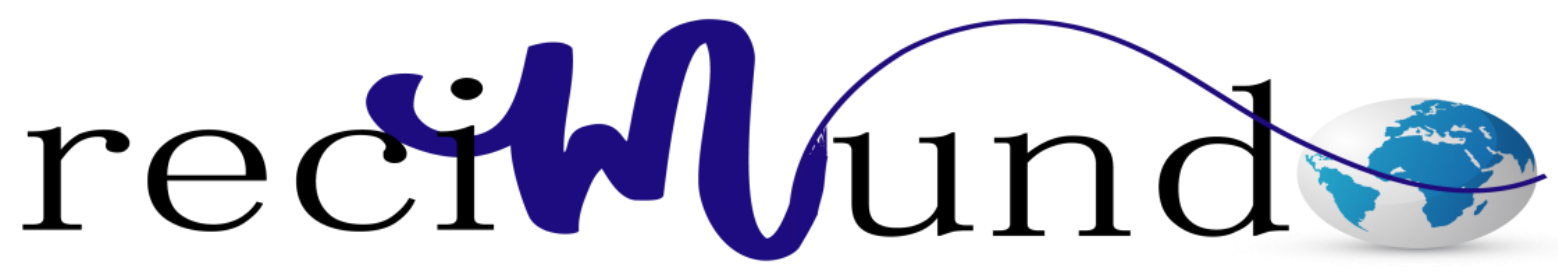

Revista Cientifica Mundo de la Investigación y el Conocimiento

José Luis Rodríguez-Matías ${ }^{\text {a; }}$ Roberto Jairo Tuesca Armijos ${ }^{\text {b; }}$ Roberto John Rueda

López $^{c}$; María Antonieta Touriz Bonifaz ${ }^{\mathrm{d}}$

La Investigación Científica en la Educación Superior

Scientific Research in Higher Education

Revista Científica Mundo de la Investigación y el Conocimiento. Vol. 2 núm.3, julio, ISSN: 2588-073X, 2018, pp. 451-464

DOI: 10.26820/recimundo/2.(3).julio.2018.451-464

Editorial Saberes del Conocimiento

Recibido: 05/04/2018

Aceptado: 15/05/2018

Publicado: 30/07/2018

Correspondencia: jose.rodriguezmat@ug.edu.ec

a. Especialista en Neurocirugia; Especialista en Cirugía General; Diploma Superior en Diseño Curricular por Competencias; Doctor en Medicina y Cirugía; Medico; Universidad de Guayaquil; jose.rodriguezmat@ug.edu.ec

b. Médico; mustangstrife@gmail.com

c. Magister en Gerencia y Administración en Salud; Doctor en Medicina y Cirugía; b2gp1@live.com

d. Magister en Epidemiologia; Magister en Salud Publica; Médica; Universidad de Guayaquil; maria.tourizb@ug.edu.ec 


\section{La Investigación Científica en la Educación Superior}

Vol. 2, núm. 3., (2018)

José Luis Rodríguez-Matías; Roberto Jairo Tuesca Armijos; Roberto John Rueda López; María Antonieta Touriz Bonifaz

\section{RESUMEN}

Estamos en la era de la información donde la educación superior y la investigación científica representan el rumbo a seguir para el desarrollo y progreso mundial. El presente trabajo se enfoca en la investigación científica en las instituciones de educación superior con énfasis en su importancia y su relación con el desarrollo económico. El estudio se realizó mediante una revisión documental bibliográfica. Algunos de los factores de cambio del mundo moderno son la globalización, la tecnología y la integración multicultural, los cuales representan una influencia positiva para el investigador. Asimismo, se estableció una relación entre la universidad y la investigación científica como una simbiosis donde una se alimenta de la otra y viceversa. Muchos de los grandes hallazgos investigativos que han cambiado el destino de la humanidad y contribuido de manera total en su desarrollo se han originado en el seno de una universidad, siendo esta institución la forjadora y copartícipe de estos cambios. Se concluyó que, dada la importancia de la investigación científica en las instituciones de educación superior, resulta fundamental el apoyo que los gobiernos de los diferentes países puedan brindar, con el objeto de conseguir un mayor desarrollo y bienestar para la humanidad.

Palabras claves: Investigación, Científica, Educación, Superior, Información. 


\title{
La Investigación Científica en la Educación Superior
}

Vol. 2, núm. 3., (2018)

José Luis Rodríguez-Matías; Roberto Jairo Tuesca Armijos; Roberto John Rueda López; María

Antonieta Touriz Bonifaz

\begin{abstract}
We are in the information age where higher education and scientific research represent the way forward for global development and progress. The present work focuses on scientific research in higher education institutions with emphasis on its importance and its relationship with economic development. The study was carried out through a bibliographic documentary review. Some of the change factors of the modern world are globalization, technology and multicultural integration, which represent a positive influence for the researcher. Likewise, a relationship between the university and scientific research was established as a symbiosis where one feeds on the other and vice versa. Many of the great research findings that have changed the destiny of humanity and contributed fully to its development have originated within a university, this institution being the forger and co-participant of these changes. It was concluded that, given the importance of scientific research in higher education institutions, the support that the governments of different countries can provide is essential, in order to achieve greater development and welfare for humanity.
\end{abstract}

Keywords: Research, Scientific, Education, Superior, Information. 


\section{La Investigación Científica en la Educación Superior}

Vol. 2, núm. 3., (2018)

José Luis Rodríguez-Matías; Roberto Jairo Tuesca Armijos; Roberto John Rueda López; María Antonieta Touriz Bonifaz

\section{Introducción.}

Akyeampong (1998) cita al Cardenal John Henry Newman, erudito del siglo XIX, quien inspirado en la máxima de Cicerón según la cual "sólo el hombre posee la capacidad de buscar y perseguir la verdad" conceptualizó la función de la universidad ideal como la búsqueda del conocimiento por sí mismo.

En este sentido, se muestra a la Universidad como centro de la búsqueda del conocimiento, motor e impulso de esta capacidad natural del hombre.

Durante el siglo XX se realizaron hallazgos históricos, descubrimientos intelectuales de la relatividad y la mecánica cuántica y la interpretación de la estructura del ADN, descubrimientos que han permitido a los investigadores aclarar la naturaleza y el comportamiento básico de algunos de sus biotipos. Estos logros, estos triunfos de la ciencia, han dado lugar a la revisión de la máxima de Newman, según la cual las instituciones de enseñanza deben buscar el conocimiento por sí mismo. (Akyeampong, 1998)

Para Puebla (2014) citando a Carlos Borsotti, 1989 "investigar es un proceso por el cual se intenta dar respuesta a problemas científicos mediante procedimientos sistemáticos, que incluyen la producción de información válida y confiable”.

Por otra parte, Peña Espinoza, (2016) la define como la manera mediante la cual el ser humano puede comprender, analizar y aprender algo nuevo o algo que desconocía y es donde radica su importancia. 


\section{La Investigación Científica en la Educación Superior}

Vol. 2, núm. 3., (2018)

José Luis Rodríguez-Matías; Roberto Jairo Tuesca Armijos; Roberto John Rueda López; María Antonieta Touriz Bonifaz

De esta forma representa un proceso sistemático que persigue la solución de un problema o sencillamente un aporte que engrandece el conocimiento humano.

Asimismo, el autor continúa indicando con respecto a la investigación científica que la universidad llega a ser el espacio para animar las capacidades transformadoras del estudiante, representa un lugar privilegiado para investigar, innovar, desarrollar el espíritu creativo y comprender qué somos, en qué universo vivimos, o en qué medida nuestra sociedad, cultura y educación, nuestra historia en el pasado y presente influyen en nuestro modo de pensar y actuar. (Peña Espinoza, 2016)

Asimismo, este espacio de estudio es fundamental y permite a los docentes y alumnos coadyuvar con sus investigaciones en el desarrollo de la humanidad.

"En la sociedad actual, la generación, apropiación y aplicación del conocimiento tienen una importancia relevante. El aprendizaje de los principios básicos del método científico es un punto de partida para la reflexión y actuación ante los fenómenos de la vida social y natural. En función a lo anterior, la práctica de investigación se convierte en una tarea ineludible”. (Torres, 2018)

El presente trabajo plasma todos los aspectos relevantes acerca de la investigación científica en la educación superior, por ser esta etapa vital para su desarrollo en la formación profesional del ser humano y por los relevantes aportes que han surgido de las diferentes universidades del mundo. 


\section{La Investigación Científica en la Educación Superior}

Vol. 2, núm. 3., (2018)

José Luis Rodríguez-Matías; Roberto Jairo Tuesca Armijos; Roberto John Rueda López; María Antonieta Touriz Bonifaz

\section{Metodología.}

La presente investigación se clasifica por el tipo de fuente como documental bibliográfica, donde se realizó una búsqueda y recuperación de temas relacionados con la investigación científica y la educación superior, resaltando su importancia, factores de cambio que tienen influencia sobre esta, su relación con el desarrollo económico, entre otros, con el fin de resaltar la influencia positiva que tiene la inversión de los países en los proyectos de investigación científica en las universidades como base del desarrollo de la humanidad.

Respecto a la metodología aplicada Arias (2012), refiere acerca de la investigación documental que "es un proceso basado en la búsqueda, recuperación, análisis, crítica e interpretación de datos secundarios, es decir, los obtenidos y registrados por otros investigadores en fuentes documentales: impresas, audiovisuales o electrónicas”.

\section{Resultados.}

Relación entre investigación y educación

La educación y la investigación tienen una relación recíproca. La investigación produce educación, esto es que educa a una sociedad y la educación potencia la investigación; ambos permiten que la persona se recree, se perfeccione, se desarrolle y se realice en el espacio-tiempo correspondiente. Es por ello, que esta correlación gesta una dimensión investigativa en la educación y que actualmente ha recobrado un auge insólito en la planificación no solo a nivel educativo sino también estatal. (Rincón, 2009) 


\section{La Investigación Científica en la Educación Superior}

Vol. 2, núm. 3., (2018)

José Luis Rodríguez-Matías; Roberto Jairo Tuesca Armijos; Roberto John Rueda López; María Antonieta Touriz Bonifaz

Esta relación representa una simbiosis donde investigación y educación se benefician una de otra, y en conjunto representan un aporte a la sociedad que plantea nuevos retos en la organización y planificación que de cada vez más impulso a la investigación dentro del sistema educativo.

Factores que inciden en la investigación científica en la educación superior

Existe una serie de factores que inciden en la forma en que se planifica, desarrolla y gestiona actualmente la investigación científica dentro de las universidades, entre los que se destacan la tecnología, la globalización y la diversidad cultural. En conjunto han influido en los cambios del mundo actual, facilitando la comunicación, la investigación, las alianzas, rompiendo barreras geográficas y alimentándonos con culturas que antes eran desconocidas, todos estos cambios han llevado a las universidades a adoptar nuevas estrategias que permitan explotar estos nuevos recursos y amplificar el alcance de las investigaciones, con la finalidad de realizar nuevos hallazgos y desarrollar al máximo el potencial tanto de docentes como estudiantes.

\section{La tecnología.}

Uno de los aspectos más resaltantes de la tecnología de la información es que resulta ser un medio sumamente útil para los investigadores. La nueva era trae la creación de la "universidad virtual", ante la cual ha algunas proyecciones indican la desaparición de la estructura de la universidad tradicional en su configuración actual. La razón de ello es que la "universidad virtual" presenta varias ventajas con respecto a la tradicional, pues fomenta la colaboración entre universidades y docentes que se encuentran en lugares alejados, permite que 


\section{La Investigación Científica en la Educación Superior}

Vol. 2, núm. 3., (2018)

José Luis Rodríguez-Matías; Roberto Jairo Tuesca Armijos; Roberto John Rueda López; María Antonieta Touriz Bonifaz

los estudiantes accedan fácilmente a bibliotecas de primera clase de distintas instituciones y constituye un medio excelente para reducir costes. (Akyeampong, 1998)

Los avances tecnológicos representan una ventaja indiscutible para los investigadores de la era actual, la automatización de los procesos y los accesos a la web permiten la búsqueda de un sinfín de fuentes, bibliotecas virtuales, ebooks, artículos, opiniones, datos estadísticos, entre otros de interés. Podemos acceder desde cualquier lugar a la información requerida para el desarrollo de una investigación, lo cual facilita el proceso y pone a disposición el material requerido en cualquier tiempo y espacio.

La ruptura de las barreras geográficas es otra ventaja del uso de las herramientas tecnológicas en la actualidad, lo cual ha fomentado las alianzas estratégicas entre universidades, creando un espíritu colaborativo reunido en pro de la investigación y la búsqueda de los mejores resultados.

Esta concepción tecnológica nacida del siglo XX dio pie a nuevos paradigmas en la estructura de la educación superior, donde la verticalidad quedó en el pasado, convirtiendo a los profesores en facilitadores y colegas y dando un mayor protagonismo al estudiante en el impulso de sus investigaciones.

\section{La globalización}

Con relación a la globalización, Sánchez (2002) refiere que "en el contexto internacional actual no solo las fronteras nacionales se vuelven porosas, también se flexibilizan las fronteras disciplinarias lo que permite, a su vez, el acercamiento entre comunidades académicas de 


\section{La Investigación Científica en la Educación Superior}

Vol. 2, núm. 3., (2018)

José Luis Rodríguez-Matías; Roberto Jairo Tuesca Armijos; Roberto John Rueda López; María

Antonieta Touriz Bonifaz

hombres y mujeres provenientes de diversas regiones del planeta. Asistimos a la intensificación de las formas de cooperación e intercambio académico, a nivel nacional e internacional. El fenómeno de la globalización puede ser una oportunidad para la realización de acuerdos y convenios entre entidades académicas de diversos países con la finalidad de tender puentes de comunicación y propiciar el establecimiento de redes, que permitan a los estudiantes y los académicos la construcción de nuevos espacios del conocimiento de carácter transdisciplinario. (p. 356-357)

En tal sentido, estando tan ligada la globalización, incluso siendo una consecuencia de la tecnología, esta viene a reforzar la teoría de la ruptura de las barreras geográficas con énfasis en las alianzas estratégicas entre las instituciones educativas de nivel superior, creando nuevas estructuras en la investigación y nuevos modelos de trabajo conjunto.

\section{La diversidad cultural.}

Por último, con respecto a la diversidad cultural Vessuri (2008) menciona el "deber de prestar atención urgente a la diversidad cultural en la educación superior y la investigación dentro del marco de la globalización. Para ello no debe aumentarse únicamente el porcentaje de grupos sociales específicos con poca representación en el campus. Por el contrario, la diversidad incluye una red de valores, políticas, prácticas, tradiciones y recursos que proporcionan mecanismos que ayudan al alumnado y al profesorado de grupos relegados o excluidos, y sirve, así como una caja de resonancia para el resto de la sociedad". (p. 125-126)

La idea del autor resalta la importancia y la influencia positiva que tiene para las universidades la inclusión de la diversidad cultural y su aporte a la investigación, reúne nuevas 


\section{La Investigación Científica en la Educación Superior}

Vol. 2, núm. 3., (2018)

José Luis Rodríguez-Matías; Roberto Jairo Tuesca Armijos; Roberto John Rueda López; María Antonieta Touriz Bonifaz

políticas, métodos, valores, políticas, las cuales enriquecen y dan un nuevo enfoque a la investigación universitaria. La globalización y la tecnología coadyuvan en el impulso de la inclusión de la diversidad cultural dentro de las universidades como elemento diferencial y enriquecedor en el desarrollo de la investigación científica.

\section{Importancia de la investigación}

"En el terreno de la producción, los servicios y la competencia la época actual se define por un cambio en el centro de gravedad de las principales fuentes de valor agregado. Los recursos intelectuales representan, hoy en día, insumos críticos para la producción de la riqueza en la medida en que se convierten en tecnología, inteligencia y consumo racional. El acceso y la aplicación al conocimiento representa una ventaja comparativa para los individuos, las empresas y las economías nacionales”. (Sánchez, 2002, p. 364)

Estamos en la era de la información y el conocimiento, donde las proyecciones estiman un reemplazo laboral del personal humano por la robótica, pasando este primero a ser trabajadores del intelecto o directores de la inteligencia artificial. En este sentido el aporte de la investigación científica es fundamental por cuanto es de donde se derivan todas estas nuevas tendencias y dictan la pauta de nuestra dirección hacia un mayor desarrollo.

"Los nuevos paradigmas que envuelven la sociedad global, redefinen el actuar de las instituciones que a ella le conciernen. Por ello, los estudiosos del desarrollo de la actividad científica de las Instituciones de Educación Superior se han visto impelidos a orientar sus investigaciones hacia el criterio de la pertinencia de su accionar frente a los cambios impuestos por el desarrollo científico, tecnológico y de innovación sin precedentes que condujo a la 


\section{La Investigación Científica en la Educación Superior}

Vol. 2, núm. 3., (2018)

José Luis Rodríguez-Matías; Roberto Jairo Tuesca Armijos; Roberto John Rueda López; María Antonieta Touriz Bonifaz

gestación de las tecnologías de información y comunicación, de cuyos alcances estas instituciones han sido protagonistas relevantes, para examinar su rol en las transformaciones epistemológicas, tecnológicas y pedagógicas en la sociedad global, en el contexto de la transdisciplinariedad y diversidad". (Colina, 2007)

Las instituciones de educación superior deben mantener su flexibilidad en la adopción de nuevas estructuras organizativas que le permitan explotar al máximo la investigación científica, debe buscar en todo momento su impulso por cuanto sus aportes han representado el desarrollo y cambio de la humanidad.

Dentro de la importancia de la investigación científica en el ámbito económico Plastino, (2008) refiere que, para inicios del siglo XXI en los EEUU, la primera economía mundial, habían 4.000 Colleges y Universidades (en el resto del mundo sólo 7.800). Solamente el Estado de California contaba con 130 universidades y sólo 14 países en el mundo para ese entonces tenían más de 130 universidades. (p. 71)

En ese sentido, podemos deducir que el apoyo a las universidades y a sus planes y proyectos de investigación es una característica propia de los países desarrollados, de donde se infiere su relación directa con el desarrollo económico de las naciones. Es notable la influencia que tiene la universidad y, por ende, el conocimiento con la economía mundial, y esto se concluye al analizar la estructura universitaria de los países más desarrollados.

En una reseña (Gómez, 2013) destaca: "El desarrollo de los países del llamado primer mundo ha radicado a través de las décadas en que han apostado por la inversión en la educación y principalmente en la investigación”. Idea esta que fortalece la importancia de la investigación 


\section{La Investigación Científica en la Educación Superior}

Vol. 2, núm. 3., (2018)

José Luis Rodríguez-Matías; Roberto Jairo Tuesca Armijos; Roberto John Rueda López; María Antonieta Touriz Bonifaz

para el desarrollo de los países y su relación con el apoyo que puedan brindar al fortalecimiento de sus programas.

Por otra parte, Arechavala (2011) cita el trabajo de Alejandro Mungaray, Jorge Ramos, Ismael Plascencia y Patricia Moctezuma, el cual explora las posibilidades de cambios institucionales en las universidades, en atención al fortalecimiento de su capacidad para contribuir al desarrollo económico por medio de la generación de conocimiento, y hacen énfasis en el carácter esencial que en el proceso tienen sus interacciones con otros actores de las redes de innovación. (Arechavala, 2011)

\section{Conclusiones.}

El desarrollo de la investigación científica en la educación superior representa un aporte sin precedentes para el desarrollo de la humanidad en general.

Factores de cambio como la globalización, la tecnología, la integración multicultural, representan una gran ventaja para el desarrollo de la investigación científica en la educación superior, cada vez son más las alianzas entre universidades, los intercambios, las nuevas herramientas tecnológicas para investigar, como parte de estos beneficios. Asimismo, se integran nuevas culturas que generan aportes importantes al conocimiento.

En el futuro una de las herramientas más valiosas para la humanidad estará constituida por la información, incluso en el mundo actual ya es usado como base de todos los procesos, en tal sentido, la investigación científica en la educación superior ve su mayor posibilidad de 


\section{La Investigación Científica en la Educación Superior}

Vol. 2, núm. 3., (2018)

José Luis Rodríguez-Matías; Roberto Jairo Tuesca Armijos; Roberto John Rueda López; María Antonieta Touriz Bonifaz

explotar al máximo este aporte: la información, resolver problemas, elaborar propuestas, entre otros.

Es en ese espacio llamado universidad donde han surgido muchos descubrimientos, por ejemplo, muchos de los avances tecnológicos han sido producidos en el seno de universidades, grandes invenciones y valiosos aportes en diferentes áreas.

Por ello es importante que los gobiernos de los diferentes países inviertan en la educación superior y en sus programas de investigación científica por cuanto es a través de ella que se ha generado innumerables aportes que permiten el desarrollo de la humanidad.

\section{Recomendaciones.}

El apoyo de las naciones al sistema de educación superior es fundamental para el desarrollo de los proyectos de investigación y por ende para los avances en los conocimientos de la humanidad, sin embargo, este ha sido durante muchos años una de los principales obstáculos presentados a nivel global, en atención a esta dificultad y tomando en cuenta la importancia reflejada en el presente estudio que tiene la investigación científica en la educación superior se recomienda:

- La elaboración de un estudio cuantitativo acerca de la relación entre el financiamiento de investigaciones científicas en instituciones de educación superior y el desarrollo de las naciones. 


\section{La Investigación Científica en la Educación Superior}

Vol. 2, núm. 3., (2018)

José Luis Rodríguez-Matías; Roberto Jairo Tuesca Armijos; Roberto John Rueda López; María Antonieta Touriz Bonifaz

\section{Referencias Bibliográficas}

Akyeampong, D. (octubre de 1998).La educación superior y la investigación: Desafíos y oportunidades. Conferencia mundial sobre la educación superior. UNESCO, París

Arechavala, R. (2011). Las universidades y el desarrollo de la investigación científica y tecnológica en México: una agenda de investigación. Revista de la Educación Superior, 40(2): 41-57. Recuperado de http://www.scielo.org.mx/pdf/resu/v40n158/v40n158a3.pdf

Arias, F. (2012). El Proyecto de Investigación. Introducción a la metodología científica. Caracas, Venezuela: Episteme.

Colina, L. (2007). La investigación en la educación superior y su aplicabilidad social Laurus. Revista de Educación, 13(25): 330-353.

Goméz, M.L. (2013). Importancia de la investigación en la universidad. El nuevo diario. Recuperado de 2018, de https://www.elnuevodiario.com.ni/opinion/291933-importanciainvestigacion-universidades/

Peña Espinoza, C. (2016). La importancia de la investigación universitaria y de incorporar la misma en el proceso formativo. Revista electrónica Universidad de Aquino Bolivia.

Plastino, A. (2008). La universidad: lugar de investigación científica y transferencia tecnológica. Capítulo 4. Educación superior y sociedad. Nueva época. Año 12, Número 1.

Puebla, A. (2014). Importancia de la investigación educativa. Consejo de Transformación Educativa.

Rincón, I. (2009). Gestión educativa para a la investigación científica y tecnológica. Gestiópolis. Recuperado de https://www.gestiopolis.com/gestion-educativa-para-la-investigacioncientifica-y-tecnologica/

Sánchez, M. (2002). La educación superior y la investigación científica: el reto del constitucionalismo del siglo XXI. Biblioteca Jurídica Virtual del Instituto de Investigaciones Jurídicas de la UNAM.

Torres, A. (2018). La importancia de la investigación educativa. Milenio. Recuperado de http://www.milenio.com/opinion/alfonso-torres-hernandez/apuntes-pedagogicos/laimportancia-de-la-investigacion-educativa

Vessuri, H. (2008). El rol de la investigación en la educación superior: implicaciones y desafíos para contribuir activamente al desarrollo humano y social. UPCommons. Portal de acceso abierto al conocimiento de la UPC. Universidad Politécnica de Catalunya. 